\title{
H II REGION METALLICITY CONSTRAINTS NEAR THE SITE OF THE STRONGLY LENSED SUPERNOVA "SN REFSDAL" AT REDSHIFT 1.49
}

\author{
Tiantian Yuan $^{1}$, Chiaki Kobayashi ${ }^{1,2}$, and Lisa J. Kewley ${ }^{1}$ \\ ${ }^{1}$ Research School of Astronomy and Astrophysics, The Australian National University, Cotter Road, Weston Creek, ACT 2611, Australia \\ ${ }^{2}$ Centre for Astrophysics Research, Science and Technology Research Institute, University of Hertfordshire, Hertfordshire AL10 9AB, UK \\ Received 2014 December 22; accepted 2015 March 12; published 2015 April 27
}

\begin{abstract}
We present the local $\mathrm{H}$ II region metallicity near the site of the recently discovered multiply lensed supernova (SN; "SN Refsdal") at redshift 1.49. "SN Refsdal" is located at the outer spiral arm $(\sim 7 \mathrm{kpc})$ of the lensed host galaxy, which we previously reported to exhibit a steep negative galactocentric metallicity gradient. Based on our updated near-infrared integral field spectroscopic data, the gas-phase metallicity averaged in an intrinsic radius of $\sim 550 \mathrm{pc}$ surrounding an $\mathrm{H}_{\text {II }}$ region $\sim 200 \mathrm{pc}$ away from the $\mathrm{SN}$ site is $12+\log (\mathrm{O} / \mathrm{H})_{\mathrm{PP} 04 \mathrm{~N} 2} \leqslant 8.67$. The metallicity averaged over nine $\mathrm{H}$ II regions at similar galactocentric distances $(\sim 5-7 \mathrm{kpc})$ as "SN Refsdal" is constrained to be $12+\log$ $(\mathrm{O} / \mathrm{H})_{\mathrm{PP} 04 \mathrm{~N} 2} \leqslant 8.11$. Given the fortuitous discovery of "SN Refsdal" in an advantageously lensed face-on spiral, this is the first observational constraint on the local metallicity environment of an SN site at redshift $z>1$.
\end{abstract}

Key words: galaxies: abundances - gravitational lensing: strong - supernovae: general

\section{INTRODUCTION}

Metallicity at the site of the supernova ( $\mathrm{SN})$ explosion provides important clues to the physical origins of the $\mathrm{SN}(\mathrm{e})$. Reciprocally, the evolution and feedback of $\mathrm{SNe}$ provide crucial recipes for the chemical enrichment models of galaxies across cosmic time (e.g., Scannapieco et al. 2006; Kobayashi et al. 2007; Few et al. 2014).

Generally, $\mathrm{SNe}$ can be attributed to either the thermonuclear explosion of a white dwarf in a binary system (Type Ia; e.g., Hillebrandt \& Niemeyer 2000; Parrent et al. 2014) or the core collapse (CC) of a massive star (Type II, Ib/c; e.g., Heger et al. 2003; Crowther 2007; Smartt 2009). Theoretically, at low metallicities $([\mathrm{Fe} / \mathrm{H}] \lesssim-1.1)$, SNe Ia may be suppressed through the single-degenerate channel as the white dwarf wind would be too weak for the mass to reach the Chandrasekhar limit (Kobayashi et al. 1998; Kobayashi \& Nomoto 2009). For $\mathrm{CC} \mathrm{SNe}$, the number ratio of $\mathrm{SNe} \mathrm{Ib} / \mathrm{c}$ to $\mathrm{SNe}$ II is expected to increase with progenitor metallicity due to the dependence of mass-loss rate on metallicity (e.g., Meynet \& Maeder 2003; Prantzos \& Boissier 2003; Eldridge 2007; Limongi \& Chieffi 2009; see also Eldridge et al. 2013; Yoon et al. 2010 for the binary scenario).

However, the strong theoretical dependence on metallicity has not been confirmed in observations. At low redshift $(z<1)$, observations have shown that the metallicity distributions of the host galaxies of $\mathrm{SNe}$ II and $\mathrm{SNe}$ Ia are statistically indistinguishable, whereas the host galaxies of $\mathrm{SN} \mathrm{Ib/c} \mathrm{favor}$ higher metallicity (e.g., Prieto et al. 2008; Modjaz et al. 2011; Stoll et al. 2013). The class of abnormally luminous SNe II/Ia favor lower-metallicity environments (e.g., Childress et al. 2011; Khan et al. 2011; Kelly \& Kirshner 2012). At high redshift $(z>1)$, measurements for SN host metallicities are still rare and deserve special attention (e.g., Frederiksen et al. 2014; Graur et al. 2014; Rodney et al. 2014).

Note that theoretical models of $\mathrm{SNe}$ are based on the metallicity of the progenitor star, whereas in observations, direct measurement for the progenitor star metallicity is only possible for $\mathrm{SNe}$ in nearby galaxies. Most studies are based on assumptions that the global metallicity of the host galaxy is a good proxy for the SNe progenitor metallicity and that gas- phase oxygen abundance is a good proxy for the stellar metallicity. Additionally, the majority of local spiral galaxies show a radial metallicity gradient, and their gradients could be significantly steeper at high redshift (e.g., Jones et al. 2010; Yuan et al. 2011). It is therefore quantitatively unclear how much systematic uncertainty in the observed metallicity and $\mathrm{SN}$-type relation is introduced by the approximation of the locally and globally averaged metallicity (e.g., Stanishev et al. 2012).

In the era of integral field spectroscopic (IFS) surveys, spatially resolved properties at the sites of $\mathrm{SNe}$ can now be measured for large samples at low redshift, enabling more accurate and unbiased local environment parameters to be probed for the SN progenitors (Galbany et al. 2014). Measuring spatially resolved metallicities at high redshift remains challenging because of the limited angular resolution and signal-to-noise (S/N; Yuan et al. 2013). With a boost of a few tens in flux and area through lensing magnification, gravitationally lensed targets are the forerunners of this field (e.g., Jones et al. 2013; Wuyts et al. 2014).

The recent discovery of a multiply lensed $\mathrm{SN}$ at $z=1.49$ ("SN Refsdal"; Kelly et al. 2014) in the cluster field of MACS J1149.5+2223 provides a rare opportunity to study the local metallicity environment of a high-redshift $\mathrm{SN}$ for the first time. In this Letter, we update the integral IFS data from Yuan et al. (2011) and place local metallicity constraints near the explosion site of "SN Refsdal." Throughout this paper, we adopt a flat cosmology with $\Omega_{M}=0.3, \Omega_{\Lambda}=0.7$, and $\mathrm{H}_{0}=70 \mathrm{~km} \mathrm{~s}^{-1} \mathrm{Mpc}^{-1}$. We use solar oxygen abundance $12+$ $\log (\mathrm{O} / \mathrm{H})_{\odot}=8.69$ (Asplund et al. 2009).

\section{NEAR-INFRARED INTEGRAL IFS DATA}

Our data were collected on the laser guide star adaptive optics (LGSAO) aided near-infrared integral field spectrograph OSIRIS (Larkin et al. 2006) at KECK II in 2010 and 2011. The data presented in Yuan et al. (2011) were taken on 2010 March 3. We obtained a total of $4.75 \mathrm{hr}$ net exposure in the Hn3 band on the lensed spiral galaxy "Sp1149" $\left(\left(\alpha_{2000}, \delta_{2000}\right)=\left(11^{\mathrm{h}} 49^{\mathrm{m}} 35^{\mathrm{s}} .284,+22^{\circ} 23^{\prime} 45^{\prime \prime} \cdot 86\right)\right)$ that is hosting "SN Refsdal." On 2011 April 22, we acquired another 

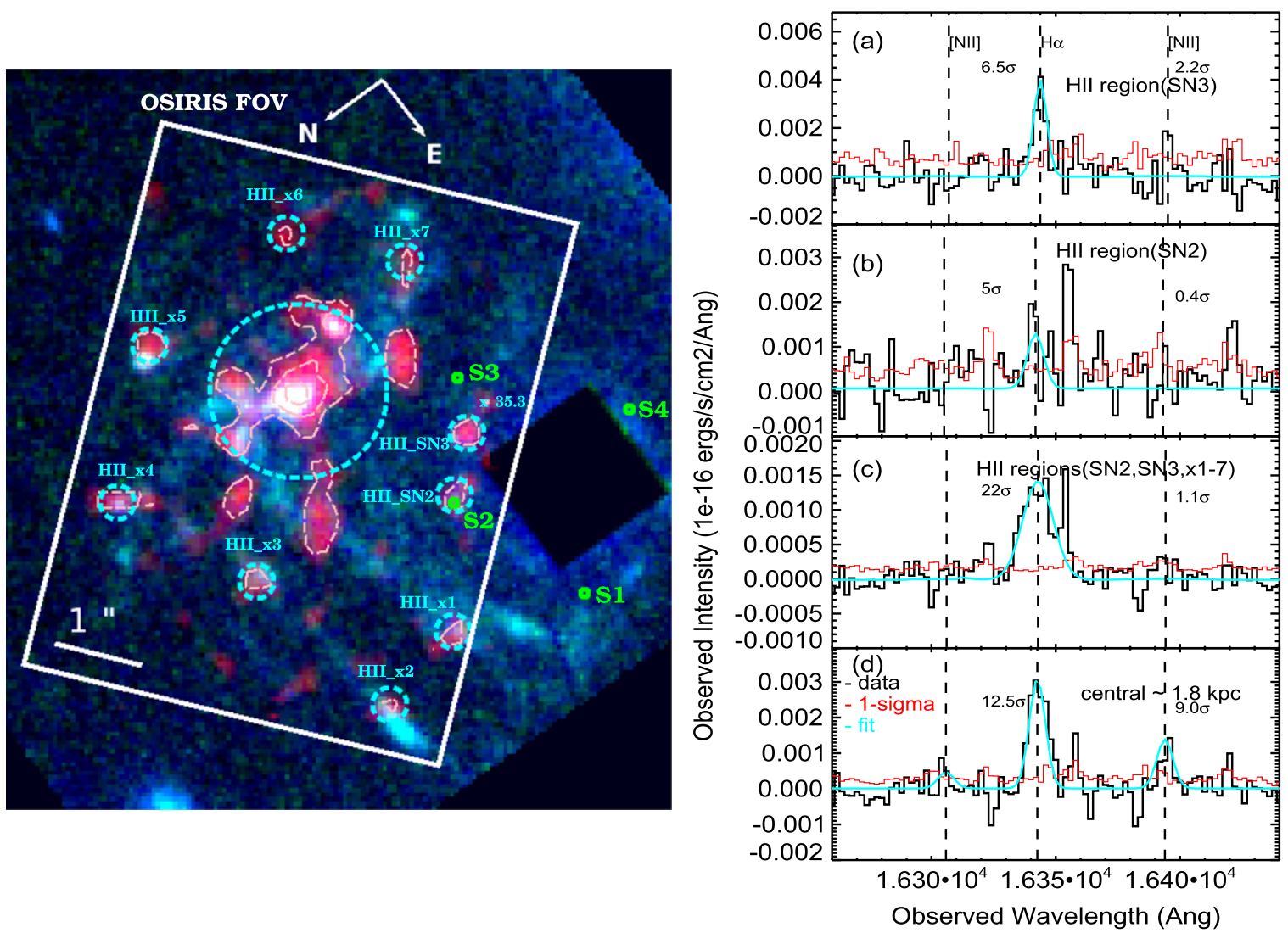

Figure 1. Left: OSIRIS H $\alpha$ (red) and HST F814W (green), F555W (blue) band color image of the host galaxy of "SN Refsdal" at $z=1.49$ behind the lensing cluster MACS J1149.5+2223. The white box shows the 4.8 by $6.4 \mathrm{arcsec}$ FOV of the OSIRIS Hn3 filter with the 0.1 per pixel plate scale. The foreground elliptical galaxy is masked out (black square). Locations of the "SN Refsdal" images are highlighted with green circles and are labeled as S1, S2, S3, and S4. Two H II regions close to S2 and S3 are labeled as "H II SN3" and "H II SN2." Five H $\alpha$ contour levels $\left(0.8,1.4,2.2,2.8\right.$, and 3.5, in units of $10^{-16} \mathrm{erg} \mathrm{s}^{-1} \mathrm{~cm}^{-2}$ arcsec $\left.{ }^{-2}\right)$ are shown in pink. One of the multiply lensed $\mathrm{H}$ in regions defined as associated with the supernova (Sharon \& Johnson 2015) is labeled as "35.3." Cyan circles indicate the nine H II regions that are at approximately similar galactocentric distances $(\sim 5-7 \mathrm{kpc})$ as "SN Refsdal." A radius of $0.3^{\prime \prime}(\sim 550 \mathrm{pc}$ on the source plane) is used to define the H II region size. Right: averaged spectra for different regions marked on the left. Raw data are shown in black; the $1 \sigma$ noise spectra are in red. Cyan lines show the best-fit Gaussian profiles for lines detected above $3 \sigma$ (S/N of the $\mathrm{H} \alpha$ and $[\mathrm{N}$ II] lines are marked).

$2 \mathrm{hr}$ of net exposure on this source with the same instrumental settings under similar weather conditions. The data were reduced using the same pipeline and analysis code as described in Yuan et al. (2011). We coadded the flux-calibrated data of 2010 and 2011 and weighted by the S/N of the total $\mathrm{H} \alpha$ flux.

To summarize, the data presented in this paper are based on a total of $6.75 \mathrm{hr}$ on-source exposure on OSIRIS Keck II. The spatial resolution in our observation is $\sim 0.1$, corresponding to a source-plane resolution of $\sim 170 \mathrm{pc}$ after correcting for a lensing magnification of $\mu_{\text {flux }}=22$ (using the same lens model as described in Yuan et al. 2011). The spectral coverage is $1.594-1.676 \mu \mathrm{m}$ with a spectral resolution of $\sim 3400$. The corresponding rest-frame wavelength range is 6406-6731 $\AA$ at the redshift of "Sp1149" $(z=1.49)$, aiming to capture the $\mathrm{H} \alpha$ and $[\mathrm{N}$ II] emission lines.

Figure 1 shows the two-dimensional $\mathrm{H} \alpha$ map from the OSIRIS datacube for $\mathrm{H} \alpha$ line detections of $\mathrm{S} / \mathrm{N} \geqslant 3 \sigma$. We use the coordinates of the SN sites on the image plane from Kelly et al. (2014) and Oguri (2015). The sites of the lensed SN images are marked with green circles ("S1-4"). Two SN locations ("S2" and "S3") are covered in the OSIRIS field of view (FOV). We detect a significant amount $(f \mathrm{H} \alpha \gtrsim$ $0.8 \times 10^{-16} \mathrm{erg} \mathrm{s}^{-1} \mathrm{~cm}^{-2} \operatorname{arcsec}^{-2}$ or $\left.\gtrsim 0.8 M_{\odot} \mathrm{yr}^{-1} \mathrm{kpc}^{-1}\right)$ of $\mathrm{H} \alpha$ emission from the $\mathrm{H}_{\text {II }}$ regions ("H II SN2" and "H II_SN3") that are close to "S2" and "S3." Most of the bright $\mathrm{H} \alpha$ knots are associated with a strong continuum, though not all continuum peaks have bright $\mathrm{H} \alpha$ emissions (Figure 1).

Note that "H II_SN2" and "H II_SN3" are not the multiply lensed $\mathrm{H}$ in regions associated with the $\mathrm{SN}$ as defined in Sharon $\&$ Johnson (2015). We detect very weak $(3 \sigma) \mathrm{H} \alpha$ emission from one of the multiply lensed $\mathrm{H}_{\text {II }}$ regions (labeled as "35.3" in Figure 1) defined in Sharon \& Johnson (2015). The spectra extracted at the location of "35.3" using the same aperture size as "H II_SN2" are similar to "H II_SN2" (see Figure 1(b)).

\section{METALLICITY}

\subsection{Oxygen Abundance $(12+\log (\mathrm{O} / \mathrm{H}))$}

We use the $[\mathrm{N}$ II $] / \mathrm{H} \alpha$ ratio and the $\mathrm{N} 2$ metallicity calibration to calculate oxygen abundance (PP04N2; linear relation of Pettini \& Pagel 2004).

In this work, we extract averaged spectra for the $\mathrm{H}$ II regions ("H II_SN3" and "H II_SN2" in Figure 1) near the SN site. We use the peak of the $\overline{\mathrm{H}} \alpha$ emission as the extraction center. A radius of $0.3(\sim 550 \mathrm{pc}$ on the source plane $)$ is chosen as the size of the $\mathrm{H}$ II regions empirically because the $\mathrm{S} / \mathrm{N}$ of the $\mathrm{H} \alpha$ line drops below three outside the radius. We test different extraction apertures, shapes, and sizes and find no statistically significant differences in the averaged spectra if the extracting aperture is centered at the peak of $\mathrm{H} \alpha$ clumps. 

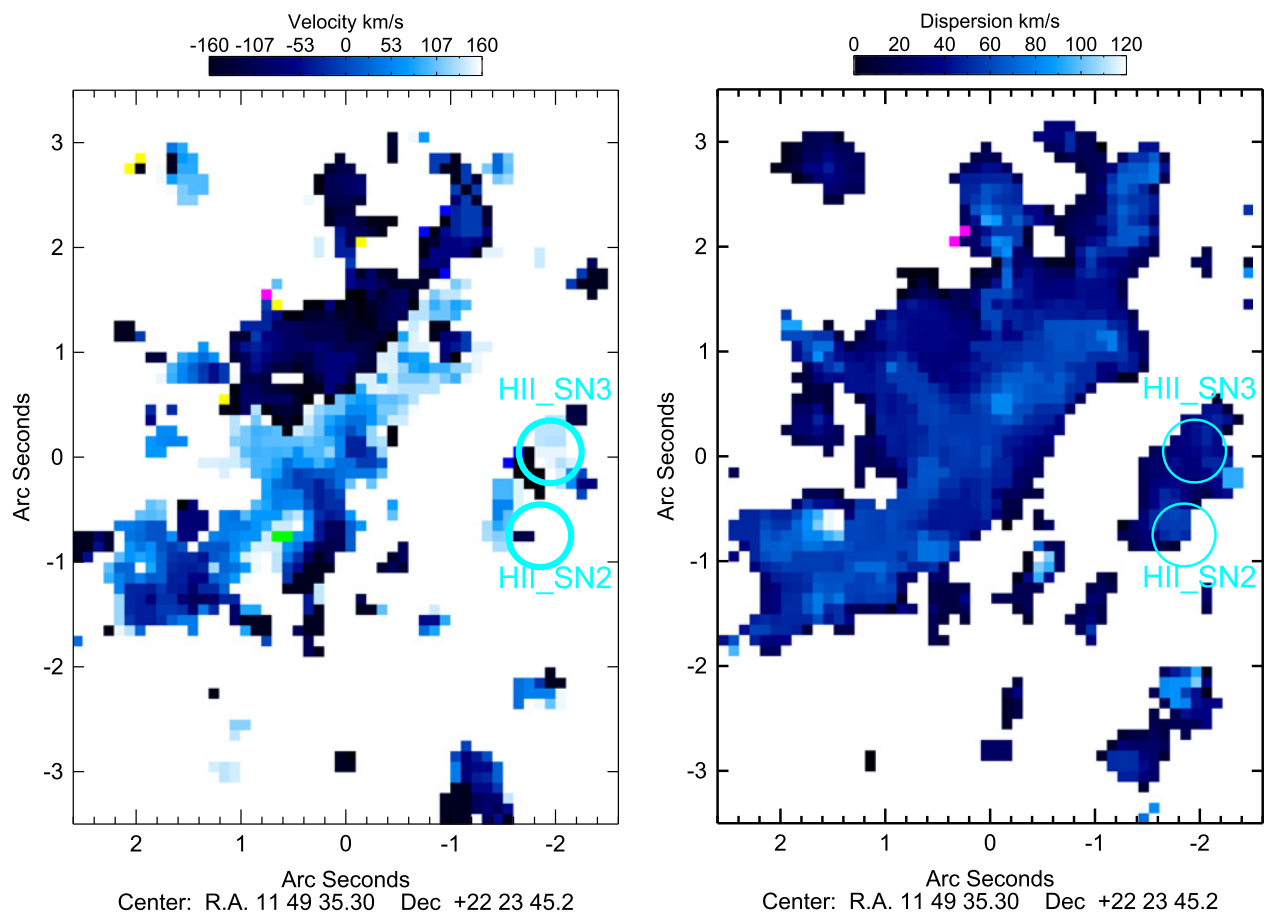

Figure 2. Velocity and velocity dispersion maps derived from $\mathrm{H} \alpha$ lines from the OSIRS datacube. Two $\mathrm{H}$ in regions that are close to the SN sites are indicated by cyan circles. There is no abnormal velocity structure or enhanced velocity dispersion indicative of strong shocks/winds at the locations of "H II_SN3" and "H II_SN2"

The $1 \sigma$ spectra in Figure 1 are derived from bootstrapping (50,000 times; weighted by $\mathrm{H} \alpha$ flux $)$ the individual spectrum of each pixel in the extraction aperture. The Gaussian line profile fitting was conducted using a $\chi^{2}$ minimization procedure with $1 / \sigma^{2}$ as the weighting. We then use the statistical error output from the line fitting procedure to estimate the $\mathrm{S} / \mathrm{N}$ of the flux. For line fluxes of $\mathrm{S} / \mathrm{N}<3$, we derive the flux upper limit by using the $1 \sigma$ spectra and median Gaussian line width $(5.27 \AA)$ of the whole galaxy. The statistical metallicity uncertainties are calculated by propagating the flux errors of the $[\mathrm{N} \mathrm{II}]$ and $\mathrm{H} \alpha$ lines. The metallicity calibration of the PP04N2 method itself has a $1 \sigma$ dispersion of 0.18 dex (Pettini \& Pagel 2004). Therefore, for individual galaxies that have statistical metallicity uncertainties of less than 0.18 dex, we use the systematic errors of 0.18 dex.

In the right panel of Figure 1, we show the averaged spectra for the $\mathrm{H}$ II regions close to the multiple images of "SN Refsdal." Unfortunately, the $[\mathrm{N}$ II $]$ line is below the $3 \sigma$ detection limit for all of the individual $\mathrm{H}$ II regions. The projected distances from the $\mathrm{H}$ II regions to the $\mathrm{SN}$ sites are $\sim 200 \mathrm{pc}$ and $1.2 \mathrm{kpc}$ for "H II_SN2 (panel (b))" and "H II_SN3 (panel (a))," respectively. "H II_SN3" is less likely to be physically associated with the SN.

To further improve the $\mathrm{S} / \mathrm{N}$ on the weak $[\mathrm{N}$ II] line, we extract a spectrum averaged across nine $\mathrm{H}$ II regions that are at similar galactocentric distances as "H II_SN2,3" (the projected distance is $\sim 5-7 \mathrm{kpc}$ on the source plane; Figure 1(c)). No $[\mathrm{N}$ II $]$ is detected above an $\mathrm{S} / \mathrm{N}$ of three in the averaged spectra across the $\mathrm{H}$ II regions, consistent with the result from Yuan et al. (2011) that $[\mathrm{N} \mathrm{II}] / \mathrm{H} \alpha$ ratios are extremely low at the outskirts of the spiral. For comparison, the average spectrum from the innermost annulus of $\sim 1.8 \mathrm{kpc}$ (source plane) is plotted at the bottom right of Figure 1.
We note that analysis on the source plane gives consistent results though the $\mathrm{S} / \mathrm{N}$ of the spectra is slightly downgraded by $1.2 \times$ due to further uncertainties in lensing models. We conclude that results based on the emission line ratios are not affected by the lensing models.

The absolute value of oxygen abundance depends on the calibration method (Kewley \& Ellison 2008). The advantages of using PP04N2 for absolute oxygen abundance include the following. (1) The $[\mathrm{N} \mathrm{III} / \mathrm{H} \alpha$ ratio is not affected by extinction, and in most cases, is the only line ratio available for highredshift observations. (2) The absolute value of PP04N2 metallicity is actually closer to direct electron temperature method (Te) metallicity than other commonly used strong line diagnostics (Kewley \& Ellison 2008). The "Te" method metallicity is considered to be a more "direct" method to calculating the absolute metallicity of galaxies, though it could be underestimating the value at very high metallicities (e.g., Nicholls et al. 2013). The caveats of using PP04N2 to diagnose the absolute oxygen abundance include the following. (1) The $[\mathrm{N} \mathrm{II}] / \mathrm{H} \alpha$ ratio is affected by ionization parameters and the presence of shocks. At a fixed metallicity, a higher ionization parameter produces lower $\left[\mathrm{N}_{\mathrm{II}}\right] / \mathrm{H} \alpha$ ratios, and the existence of shock will elevate $[\mathrm{N}$ II $] / \mathrm{H} \alpha$ ratios (e.g., Rich et al. 2011; Kewley et al. 2013; Morales-Luis et al. 2013). (2) The N/O ratio may vary across galaxies, and nitrogen fails to be a good proxy for oxygen abundance when the primary nitrogen production is dominating (e.g., Morales-Luis et al. 2013). The N2 index should not be used at very low oxygen abundances because the above caveats are most severe at low metallicities $(12+\log (\mathrm{O} / \mathrm{H}) \lesssim 8.0$; Morales-Luis et al. 2013).

Keeping the caveats in mind, the upper limit we derived for the $\mathrm{SN}$ site is still useful as a guide. The $\mathrm{H}$ II region (H II_SN2) that is $\sim 200$ pc away from the SN has an upper limit metallicity of $12+\log (\mathrm{O} / \mathrm{H}) \leqslant 8.67$. Furthermore, the upper limit of $12+\log (\mathrm{O} / \mathrm{H}) \leqslant 8.11$ from nine $\mathrm{H}$ II regions implies that the 
Table 1

Metallicity and Observed Line Fluxes

\begin{tabular}{|c|c|c|c|c|}
\hline $\begin{array}{l}\text { Region } \\
\text { (1) }\end{array}$ & $\begin{array}{l}\text { Flux } \mathrm{H} \alpha \\
\quad(2)\end{array}$ & $\begin{array}{c}\text { Flux }[\mathrm{N} \text { II] } \\
\quad(3)\end{array}$ & $\begin{array}{c}12+\log (\mathrm{O} / \mathrm{H}) \mathrm{PP} 04 \mathrm{~N} 2 \\
(4)\end{array}$ & $\begin{array}{c}{[\mathrm{Fe} / \mathrm{H}]} \\
(5)\end{array}$ \\
\hline $\mathrm{H}_{\text {II }} \mathrm{SN} 3\left(1.2 \mathrm{kpc}\right.$ from $\left.\mathrm{SN}^{\mathrm{a}}\right)$ & $0.026 \pm 0.004$ & $\leqslant 0.004$ & $\leqslant 8.45$ & $\leqslant-0.64$ \\
\hline H ІІ SN2 (200 pc from SN) & $0.010 \pm 0.002$ & $\leqslant 0.004$ & $\leqslant 8.67$ & $\leqslant-0.36$ \\
\hline H II SN2,3, H II x1-7 (5-7 kpc galactocentric) & $0.022 \pm 0.001$ & $\leqslant 0.0009$ & $\leqslant 8.11$ & $\leqslant-1.06$ \\
\hline Central $1.8 \mathrm{kpc}$ & $0.025 \pm 0.002$ & $0.011 \pm 0.0012$ & $8.69 \pm 0.05^{\mathrm{b}}$ & -0.34 \\
\hline Whole galaxy ${ }^{\mathrm{c}}$ & $0.024 \pm 0.0008$ & $0.004 \pm 0.001$ & $8.43 \pm 0.05^{\mathrm{b}}$ & -0.66 \\
\hline
\end{tabular}

Notes. (1) Region where the spectra are averaged, labeled in Figure 1. (2), (3) Averaged flux density over respective spatial regions; in units of $10^{-16} \mathrm{erg} \mathrm{s}^{-1} \mathrm{~cm}^{-2}$ per spaxel $\left(0,1^{\prime}\right)$. $1 \sigma$ upper limit is derived for line detection below an $\mathrm{S} / \mathrm{N}$ of five. (4) Metallicity based on the PP04N2 method. (5) [Fe/H] inferred from oxygen abundance (see Section 3.2)

${ }^{a}$ Intrinsic distance corrected for lensing, uncorrected for orientation. Typical uncertainty of the distance from lens model is $\sim 20 \%$.

${ }^{b}$ This is the statistical error. Systematic error from the N2-dex method is $\sim 0.18$ dex.

${ }^{\mathrm{c}}$ The average flux density of the whole galaxy is derived using the same method as the $\mathrm{H}$ II regions, with an aperture size that includes the whole galaxy. The total SFR of the galaxy after lensing and dust correction is $\sim 1.2 \mathrm{M}_{\odot} \mathrm{yr}^{-1}$ (Livermore et al. 2015)

oxygen abundance is extremely low: more than $\sim 0.5$ dex lower than the central $1.8 \mathrm{kpc}$ and $\sim 0.3 \mathrm{dex}$ lower than the average metallicity of the whole galaxy. We can not quantify the effect of shock or variations in ionization parameters. However, we do not find enhanced velocity dispersion indicative of strong shocks/winds at the locations of "H ${ }_{\text {II }}$ SN3" and "H II SN2" (see Figure 2).

\subsection{Iron Abundance $[\mathrm{Fe} / \mathrm{H}]$}

Because it is iron rather than oxygen that provides the dominant opacity for the white dwarf winds in the late stage of stellar evolution (Kobayashi et al. 1998), we infer stellar iron abundance $([\mathrm{Fe} / \mathrm{H}])$ using gas-phase oxygen abundance $(12+\log (\mathrm{O} / \mathrm{H}))$.

The $[\mathrm{O} / \mathrm{Fe}]$ ratios have a well-known relation with $[\mathrm{Fe} / \mathrm{H}]$ in the solar neighborhood, where $[\mathrm{O} / \mathrm{Fe}]$ is constant at $[\mathrm{Fe} / \mathrm{H}] \lesssim-1$ and linearly decreases at $[\mathrm{Fe} / \mathrm{H}] \gtrsim-1$ (e.g., Cayrel et al. 2004). The plateau $[\mathrm{Fe} / \mathrm{H}]$ is determined from the IMF weighted yields of CC SNe (SNII and Ibc) and is 0.45 , consistent with observations of stars with 3D/NLTE corrections (Kobayashi et al. 2006). With this constant, the upper limit of $[\mathrm{Fe} / \mathrm{H}]$ near the site of "SN Refsdal" is -1.06 for $12+\log (\mathrm{O} / \mathrm{H}) \leqslant 8.11(9$ $\mathrm{H}$ II average).

We use the prescriptions in Stoll et al. (2013) for the conversion: $[\mathrm{Fe} / \mathrm{H}]=-11.2+1.25(12+\log (\mathrm{O} / \mathrm{H}))_{\mathrm{PP} 04 \mathrm{~N} 2}$. We list the converted $[\mathrm{Fe} / \mathrm{H}]$ values for $\mathrm{SN}$ sites in Table 1 . Here, we adopt the solar metallicity $12+\log (\mathrm{O} / \mathrm{H})=8.69$ (Asplund et al. 2009). If we use other values of solar metallicity, e.g., $12+\log (\mathrm{O} / \mathrm{H})=8.86$ from Delahaye \& Pinsonneault (2006), the $[\mathrm{Fe} / \mathrm{H}]$ ratio is $\sim 0.2$ dex lower. This conversion is based on fitting to the linear correlation between $[\mathrm{Fe} / \mathrm{H}]$ and $[\mathrm{O} / \mathrm{H}]$, which is valid above $[\mathrm{Fe} / \mathrm{H}]=-1$.

Note that oxygen is one of the $\alpha$ elements $(\mathrm{O}, \mathrm{Mg}, \mathrm{Si}, \mathrm{S}$, and $\mathrm{Ca})$, and observed $[\alpha / \mathrm{Fe}]$ ratios do depend on the system; $[\alpha /$ $\mathrm{Fe}]$ is higher at the given $[\mathrm{Fe} / \mathrm{H}]$ in the Galactic bulge and thick disk, while $[\alpha / \mathrm{Fe}]$ is lower at the given $[\mathrm{Fe} / \mathrm{H}]$ in Magellanic Clouds and dwarf spheroidal galaxies (e.g., Kobayashi et al. 2006). Damped Ly $\alpha$ systems, which may correspond to the outskirts of spiral galaxies at high redshifts, show low $[\alpha /$ $\mathrm{Fe}]$ ratios, although this depends on the dust depletion correction (e.g., Wolfe et al. 2005). This $[\mathrm{O} / \mathrm{Fe}]$ variation is not taken into account in column 5 of Table 1 . It is unknown if the relation between $\mathrm{O}$ and $\mathrm{Fe}$ stellar abundances can be used to

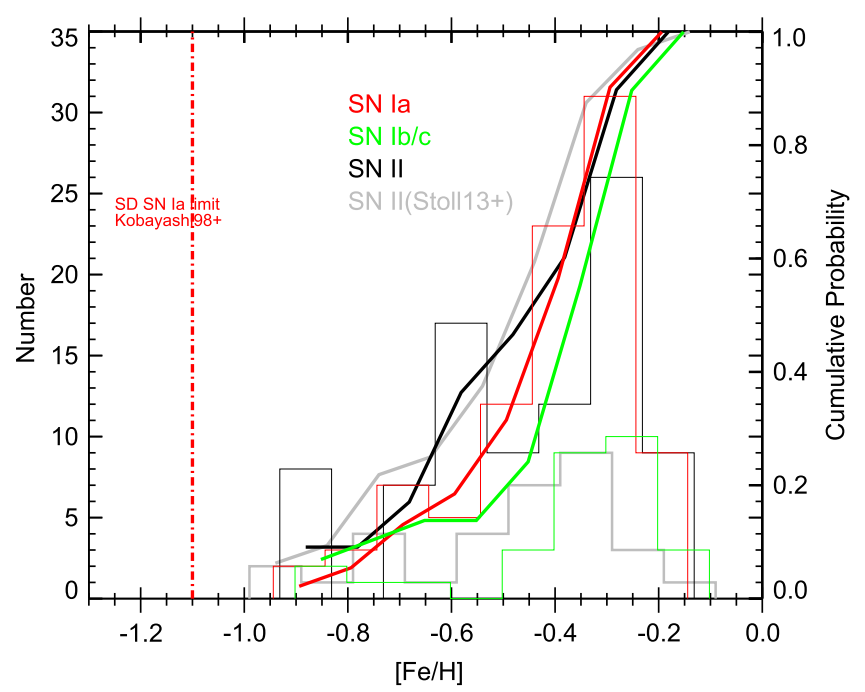

Figure 3. Histogram and cumulative distribution of $[\mathrm{Fe} / \mathrm{H}]$ for the $\mathrm{SNe}$ Ia, II, $\mathrm{Ib} / \mathrm{c}$ sample from Prieto et al. (2008) and SNe II from Stoll et al. (2013). These local samples show that $\mathrm{SNe} \mathrm{Ib} / \mathrm{c}$ occur in higher-metallicity host galaxies than $\mathrm{SNe}$ II, whereas there is no statistically significant difference between SNe Ia and SNe II. The KS probability tests of two host metallicity samples being drawn from the same distribution are 5\% (II-Ib/c), 3\% (Ia-Ib/c), and 56\% (IIIa). The vertical dashed-dotted line highlights the theoretical $[\mathrm{Fe} / \mathrm{H}]$ limit of $\lesssim-1.1$, below which the single-degenerate path $\mathrm{SN}$ Ia is forbidden for $\widetilde{\mathrm{M}}_{W D}=1 M \odot($ Kobayashi et al. 1998).

convert the gas abundance. We therefore caution the limitation of this method.

\section{DISCUSSION}

Current observational studies on local galaxies have shown some patterns between the types of SN progenitors and the host galaxy metallicities. For example, by directly comparing the central oxygen abundance of the hosts of $\mathrm{SNe} \mathrm{Ib} / \mathrm{c}$ and $\mathrm{SNe} \mathrm{Ia}$ with SNe II in SDSS galaxies, Prieto et al. (2008) found strong evidence that $\mathrm{SNe} \mathrm{Ib} / \mathrm{c}$ occur in higher-metallicity host galaxies than $\mathrm{SNe}$ II, whereas there is no statistically significant difference between SNe Ia and SNe II. Similar conclusions are also found in other studies that have metallicities directly measured at the SN sites (Modjaz et al. 2008; Stoll et al. 2013). Although normal SNe Ia have not been found at $[\mathrm{Fe} / \mathrm{H}] \lesssim-1$, the metallicity of one of the most metal-poor SN Ia host 
galaxies is $12+\log (\mathrm{O} / \mathrm{H})=8.01$ (with the R23 method; Childress et al. 2011).

To illustrate this, in Figure 3 we plot the histogram and cumulative distribution of $[\mathrm{Fe} / \mathrm{H}]$ for the $\mathrm{SNe} \mathrm{Ia}, \mathrm{II}, \mathrm{Ib} / \mathrm{c}$ sample from Prieto et al. (2008). We derive the oxygen abundance using PP04N2 and convert it to $[\mathrm{Fe} / \mathrm{H}]$ using the same methods described in Section 3.2. We also plot the sample of Stoll et al. (2013) for Type II SN progenitor regions from a single-source survey that minimized the host galaxy selection bias. The metallicity distributions of $\mathrm{SNe}$ II from the two samples agree well, and there is no distinguishable difference between Types Ia and II. Types Ib/c show a preference toward higher $[\mathrm{Fe} / \mathrm{H}]$.

The vertical dashed-dotted line in Figure 3 marks the theoretical $[\mathrm{Fe} / \mathrm{H}]$ limit of $\sim-1.1$, below which the singledegenerate path SN Ia is forbidden (Kobayashi et al. 1998). Observations have shown evidence that such a boundary for SNe Ia exists. For example, Rodney et al. (2014) found a low rate of $\mathrm{SNe}$ Ia at $z>1$, which could be explained by a population of single-degenerate-dominated Type Ia at high redshift. Note that certain types of SNe may occur only for lowmetallicity progenitor stars (e.g., Langer et al. 2007). Childress et al. (2011) found super-Chandrasekhar candidates in metalpoor environments.

The inferred $[\mathrm{Fe} / \mathrm{H}]$ upper limit of $\lesssim-1$ using the nine $\mathrm{H}$ II region average is close to the boundaries of the Kobayashi et al. (1998) limit, causing mild tension for "SN Refsdal" to be a normal Type Ia. The $[\mathrm{Fe} / \mathrm{H}]$ of "SN Refsdal" is at the low end tail of the $[\mathrm{Fe} / \mathrm{H}]$ distribution of local SNe. Given that Type $\mathrm{Ib} / \mathrm{c}$ usually favor high-metallicity environments, it could imply that "SN Refsdal" is less likely to be a $\mathrm{Ib} / \mathrm{c}$ in a relative sense. However, the SN type of "SN Refsdal" is still unknown. Future follow-up observations will determine the SN type of "SN Refsdal" and thus lead to more meaningful discussion on the metallicity constraints.

\section{CONCLUSION}

We use our updated near-infrared integral IFS data to place constraints on the local metallicity near the site of "SN Refsdal." Based on our updated near-infrared integral IFS data, the gas-phase metallicity averaged in an intrinsic radius of $\sim 550 \mathrm{pc}$ surrounding an $\mathrm{H}$ II region $\sim 200 \mathrm{pc}$ away from the $\mathrm{SN}$ site is $12+\log (\mathrm{O} / \mathrm{H})_{\mathrm{PP} 04 \mathrm{~N} 2} \leqslant 8.67$. The metallicity averaged over nine $\mathrm{H}$ II regions at similar galactocentric distances $(\sim 5-7 \mathrm{kpc})$ as "SN Refsdal" is constrained to be $12+$ $\log (\mathrm{O} / \mathrm{H})_{\mathrm{PP} 04 \mathrm{~N} 2} \leqslant 8.11$.

Given the fortuitous discovery of "SN Refsdal" in an advantageously lensed face-on spiral, this is by far the best observational constraint we can set on the local metallicity environment of an SN site at redshift $z>1$. The next generation of $30 \mathrm{~m}$ telescopes, such as TMT and GMT, are ideal for pursuing the $\mathrm{H}$ II region metallicity of the host galaxy of "SN Refsdal." Future spectroscopic and imaging follow-up work is required to conclude the SN type of "SN Refsdal." Our metallicity constraints provide an important parameter to understand the physical origin of "SN Refsdal."

We thank the referee for insightful criticism that has significantly improved this paper. T.Y. thanks Mike Childress, Fang Yuan, Richard Scalzo, and D.-C. Nicholls for useful discussion. L.K. acknowledges the support of an ARC Future
Fellowship and ARC Discovery Project DP130103925. The data presented in this paper were obtained at the W.M. Keck Observatory. The authors wish to recognize and acknowledge the very significant cultural role and reverence that the summit of Mauna Kea has always had within the indigenous Hawaiian community.

\section{REFERENCES}

Asplund, M., Grevesse, N., Sauval, A. J., \& Scott, P. 2009, ARA\&A, 47, 481 Cayrel, R., Depagne, E., Spite, M., et al. 2004, A\&A, 416, 1117

Childress, M., Aldering, G., Aragon, C., et al. 2011, ApJ, 733, 3 Crowther, P. A. 2007, ARA\&A, 45, 177

Delahaye, F., \& Pinsonneault, M. H. 2006, ApJ, 649, 529

Eldridge, J. J. 2007, RMxAA Conf. Ser., 30, 35

Eldridge, J. J., Fraser, M., Smartt, S. J., Maund, J. R., \& Crockett, R. M. 2013, MNRAS, 436, 774

Few, C. G., Courty, S., Gibson, B. K., Michel-Dansac, L., \& Calura, F. 2014, MNRAS, 444, 3845

Frederiksen, T. F., Graur, O., Hjorth, J., Maoz, D., \& Poznanski, D. 2014, A\&A, 563, A140

Galbany, L., Stanishev, V., Mourão, A. M., et al. 2014, A\&A, 572, A38

Graur, O., Rodney, S. A., Maoz, D., et al. 2014, ApJ, 783, 28

Heger, A., Fryer, C. L., Woosley, S. E., Langer, N., \& Hartmann, D. H. 2003, ApJ, 591, 288

Hillebrandt, W., \& Niemeyer, J. C. 2000, ARA\&A, 38, 191

Jones, T., Ellis, R., Jullo, E., \& Richard, J. 2010, ApJL, 725, L176

Jones, T., Ellis, R. S., Richard, J., \& Jullo, E. 2013, ApJ, 765, 48

Kelly, P. L., \& Kirshner, R. P. 2012, ApJ, 759, 107

Kelly, P. L., Rodney, S. A., Treu, T., et al. 2014

Kewley, L. J., Dopita, M. A., Leitherer, C., et al. 2013, ApJ, 774, 100

Kewley, L. J., \& Ellison, S. L. 2008, ApJ, 681, 1183

Khan, R., Stanek, K. Z., Stoll, R., \& Prieto, J. L. 2011, ApJL, 737, L24

Kobayashi, C., \& Nomoto, K. 2009, ApJ, 707, 1466

Kobayashi, C., Springel, V., \& White, S. D. M. 2007, MNRAS, 376, 1465

Kobayashi, C., Tsujimoto, T., Nomoto, K., Hachisu, I., \& Kato, M. 1998, ApJL, 503, L155

Kobayashi, C., Umeda, H., Nomoto, K., Tominaga, N., \& Ohkubo, T. 2006, ApJ, 653, 1145

Langer, N., Norman, C. A., de Koter, A., et al. 2007, A\&A, 475, L19

Larkin, J., Barczys, M., Krabbe, A., et al. 2006, NewAR, 50, 362

Limongi, M., \& Chieffi, A. 2009, MmSAI, 80, 151

Livermore, R. C., Jones, T., Richard, J., et al. 2015, arXiv:1503.07873

Meynet, G., \& Maeder, A. 2003, A\&A, 404, 975

Modjaz, M., Kewley, L., Bloom, J. S., et al. 2011, ApJL, 731, L4

Modjaz, M., Kewley, L., Kirschner, R. P., et al. 2008, AJ, 135, 1136

Morales-Luis, A. B., Sánchez Almeida, J., Pérez Montero, E., et al. 2013, in Highlights of Spanish Astrophysics VII Proc. of X Scientific Meeting of Spanish Astronomical Society (SEA), ed. J. C. Guirado, L. M. Lara, V. Quilis, \& J. Gorgas, 294

Nicholls, D. C., Dopita, M. A., Sutherland, R. S., Kewley, L. J., \& Palay, E. 2013, ApJS, 207, 21

Oguri, M. 2015, MNRAS, 449, L86

Parrent, J., Friesen, B., \& Parthasarathy, M. 2014, Ap\&SS, 351, 1

Pettini, M., \& Pagel, B. E. J. 2004, MNRAS, 348, L59

Prantzos, N., \& Boissier, S. 2003, A\&A, 406, 259

Prieto, J. L., Stanek, K. Z., \& Beacom, J. F. 2008, ApJ, 673, 999

Rich, J. A., Kewley, L. J., \& Dopita, M. A. 2011, ApJ, 734, 87

Rodney, S. A., Riess, A. G., Strogler, L.-G., et al. 2014, AJ, 148, 13

Scannapieco, C., Tissera, P. B., White, S. D. M., \& Springel, V. 2006, MNRAS, 371, 1125

Sharon, K., \& Johnson, T. L. 2015, ApJL, 800, L26

Smartt, S. J. 2009, ARA\&A, 47, 63

Stanishev, V., Rodrigues, M., Mourão, A., \& Flores, H. 2012, A\&A, 545, A58 Stoll, R., Prieto, J. L., Stanek, K. Z., \& Pogge, R. W. 2013, ApJ, 773, 12 Wolfe, A. M., Gawiser, E., \& Prochaska, J. X. 2005, ARA\&A, 43, 861 Wuyts, E., Rigby, J. R., Gladders, M. D., \& Sharon, K. 2014, ApJ, 781, 61 Yoon, S.-C., Woosley, S. E., \& Langer, N. 2010, ApJ, 725, 940

Yuan, T.-T., Kewley, L. J., \& Rich, J. 2013, ApJ, 767, 106

Yuan, T.-T., Kewley, L. J., Swinbank, A. M., Richard, J., \& Livermore, R. C. 2011, ApJL, 732, 106 